\title{
Manipulation of biotic signaling: a new theory for smarter pest control
}

\author{
Wei Qian ${ }^{1}$, Xiaoya Chen ${ }^{2}$, Rongxiang Fang ${ }^{1} \&$ Le Kang $^{3 *}$ \\ ${ }^{1}$ State Key Laboratory of Plant Genomics, Institute of Microbiology, Chinese Academy of Sciences, Beijing 100101, China; \\ ${ }^{2}$ State Key Laboratory of Plant Molecular Genetics, Institute of Plant Physiology and Ecology, Chinese Academy of Sciences, \\ Shanghai 200032, China; \\ ${ }^{3}$ State Key Laboratory of Integrated Management of Pest Insects and Rodents, Institute of Zoology, Chinese Academy of Sciences, \\ Beijing 100101, China
}

Received July 26, 2017; published online July 28, 2017

Citation: Qian, W., Chen, X., Fang, R., and Kang, L. (2017). Manipulation of biotic information: a new theory for smarter pest control. Sci China Life Sci 60, 781-784. doi: 10.1007/s11427-017-9148-X

Modern agricultural bio-technologies have significantly increased the efficiency and quality of crop production and contributed to the improvement of people's life in the world. Widespread utilization of fertilizers, herbicides, fungicides, pesticides, antibiotics, and plant growth regulators has been instrumental in securing crop yields to feed over seven billions of human beings in the earth. However, during the recent half century, extensive uses of pesticides and other chemicals, which traditionally target the essential physiological pathways of pathogenic microbes and pests, have proverbial, negative impacts on the environmental and human health. Therefore, the urgent demands for sustainable agriculture rely on a better understanding of interactions between pants and other organisms and the underpinning molecular metabolisms.

In the recent three decades, great progress has been made in understanding the relationship between plants and other organisms. Now scientists have established the notion that communication within and among species in communities is mainly mediated by infochemicals, including secondary metabolites and proteins. For example, plant secondary metabolites are essential in defense against the infection or attacks by bacteria, fungi, insects, and other herbivores. Plants also integrate chemical signals produced by the attackers to respond appropriately. On the other side of the frontline, plant pathogens manipulate host defenses by se-

*Corresponding author (email: lkang@ioz.ac.cn) creting virulence factors and even effectors that are directly injected into plant cells. Rather than acting as passive victims in these interactions, plants respond to both conserved and variable pathogen elicitors. In addition, infections by vector-borne pathogens are a major source of emerging diseases of plants. The ability of vectors to bridge spatial and ecological gaps between pathogens (especially virus) and plants increases opportunities for infection. Small adaptations of a pathogen to a vector can have profound effects on the rate of transmission. In addition, insect pests can also be attacked and killed by an array of entomopathogenic microorganisms. Unraveling these complex interactions among plants, microbial pathogens and insects holds promise for developing novel strategies to protect crop plants without compromising agricultural productivity and environmental health. Needless to say, cooperation of scientists of different disciplines is necessary to challenge these questions.

In the year of 2014, Chinese Academy of Sciences (CAS) sponsored a five-year research project, named "Decoding bio-interactions and mechanism for target management of agricultural pests" to systematically investigate the relationship between crops and other organisms. Under the umbrella of the project, 36 independent labs from seven CAS institutes are integrated into a new research team, leading to the biggest and strongest multi-disciplinary research program in biotic interaction research in China. The scientists collaborate with each other to elucidate the biotic information with multi-disciplinary studies in plant biology, microbiology, bioinformatics, genomics, and entomology at 
the molecular, organismal, and agriecosystem levels. The long-term goal of this research program is the integrated and comprehensive understanding of crop interactions with other organisms and to provide insights for creative approaches to agricultural and environmental pest control. The specific objectives of the program are: (i) to investigate the molecular, chemical and neuronal bases of phytophagous pest behaviors; (ii) to study the mechanisms of plant defense responses against insects and microbes; (iii) to understand the microbial pathogenesis of phytopathogenic bacteria and fungi; (iv) to detect the symbiotic and pathogenic interactions of insect pests with insect-associated microbes and viruses; (v) to understand the molecular and chemical mechanisms and effect of environmental changes on multi-trophic communications; (vi) to develop RNAi and/or CRISPR/Cas9 technologies as well as organic chemical and small compounds to specific pest control based on target molecules. To date, scientists in this program have made great progresses and many of their achievements have been published in the top-tier academic journals. For example, for the first time in the scientific community, genome editing technology was employed to successfully modify the complex genomes of rice and wheat to obtain disease resistance plants; We also found that plant inject their small non-coding RNA into pathogenic fungi to manipulate the latter's physiological process. In addition, a number of important components of plant innate immune system or virulence factors of pathogens were identified, which is crucial to understand the molecular mechanism of plant to defend microbial pathogens such as bacteria, fungi and virus.

This thematic issue of Science China Life Sciences presents a collection of research progresses of the abovementioned project. It contains a total of 17 reviews, research articles or letters to Editors associated to the topic of plant-pest interactions. One of the research hot spots in plant immunity is the identification of plant pattern recognition receptors (PRRs) that recognize pathogen/ microbe-associated molecular patterns (PAMPs/MAMPs). Sun et al. systematically reviewed the advances in this area, with special reference to PRRs acting as decoy proteins to sense virulence effectors of pathogens. Study in these PRRs may expand the breeding of plant resistance using artificially designed decoys in breeding (Sun et al., 2017). Xiao et al found that abscisic acid (ABA), an important plant hormone, negatively controls disease resistance of plant to prevent the post-penetration of powdery mildew fungus (Xiao et al., 2017). Since ABA plays a critical role in modulating senility of plants, this result emphasizes a relationship between plant development and disease resistance.

Verticillium dahlia is a devastating pathogenic fungus to infect cotton and cause serious yield loss. For lacking of source of resistance, how to control this pathogen is a long-term problem in agriculture. Wang et al found that overexpression of a cotton profilin gene GhPFN2 not only reorganized actin architecture of plant, but also enhanced the capability to resist to V. dahlia (Wang et al., 2017). This work thus implies that we can promote the quality of cotton and reinforce disease resistance simultaneously by manipulating the cellular cytoskeleton system. In addition, $\mathrm{Du}$ et al. estimated the expression levels of pathogenesis-related protein genes of cotton under the treatment of multiple PAMPs, such as flg22 and nlp20, these genes can be used as molecular markers in studying the immune response of cotton during resistance to the Verticillium wilt disease (Du et al., 2017).

Aphids are a large group of insects that caused serious agricultural losses by directly feeding on plant and transmitting vector-borne virus. However, our knowledge on aphids are lacking. Guo et al. reported the transcriptomic difference of three aphis species under the insecticide stress conditions, they found that expressions of genes involved in hydrolase activity, calcium ion binding, transportation and detoxification were remarkably changed, representing the cellular responses of aphid in resisting insecticide (Guo et al., 2017). Zhang et al. determined the activities of detoxification enzymes of aphid populations and found that evolutionary conserved enzymes in different species of aphids, suggesting that these proteins are critical in resisting to insecticide (Zhang et al., 2017). In addition, the green mirid bug Apolygus lucorum is an agricultural pest that is known to cause damage to more than 150 plant species, however, biology of this pest is also less studied. Chen et al. generated a high-quality transcriptomes of Apolygus lucorum for further characterization of this emerging and important pest (Chen et al., 2017). Zhang et al. studied the immune responses of a vector beetle, Monochamus alternatus, when infected by entomopathogenic fungus Beauveria bassiana. They found that Toll and IMD pathways played a leading role in the beetle's immune system when infected by the fungus. In another research article (Zhang et al., 2017), Zhang et al reported a result that there is developmental difference of pinewood nematode Bursaphelenchus xylophilus, which may be associated with the infectious process (Zhang et al., 2017). The significance of these findings is by no means restricted in the experimental conditions. For example, climate change has profound effect on the behavior of insects. Guo et al. reviewed that the progress in studying the biological significance of elevated $\mathrm{CO}_{2}$ or $\mathrm{O}_{3}$ in regulating vector insects and plant viruses during infection of host plants. They discussed that phytohormones, such as salicylates and jasmonates, play essential roles in plants reactions to elevated $\mathrm{CO}_{2}$ or $\mathrm{O}_{3}$, and thus indirectly affecting the interactions between insect and plant (Guo et al., 2017).

Inter-kingdom signaling between different organisms is a fascinating research areas in biology. Kan et al. reviewed the current progress of inter-kingdom communications between plants and microbes, with special emphasis on the quorum sensing signals eliciting bacterial and host reactions 
(Kan et al., 2017). In a Letter to Editor, Wang et al. reported a number of volatiles generated by bacteria that affect the antagonistic effect of symbiotic fungus, suggesting that bacterial symbionts of insects indirectly benefit hosts by inhibiting the growth of detrimental fungus (Wang et al., 2017). Besides inter-kingdom signaling, the molecular mechanism of pathogenic microbes is also a topic in this special issue. For example, rice blast caused by the pathogenic fungus Magnaporthe oryzae (M. oryzae) is one of the most destructive diseases worldwide. Cao et al. re-sequenced two field isolates of the fungus and compared the genomes with reference sequences. They identified important genetic polymorphic loci that have potential impacts on the virulence and rapid evolution of this notorious pathogen (Cao et al., 2017). Lai et al. used RNA-seq approach to analyze the gene expression profiling of Beauveria bassiana that infects mosquitoes. Stage-specific genes and genes involved in infecting insect host were obtained from the investigation, which facilitates understanding of $B$. bassiana pathogenesis and its interaction with mosquito. We anticipated that application of entomopathogenic fungi to control mosquitoes is a promising tool for reducing vector-borne disease transmission (Lai et al., 2017). Zhang et al. identified a polyketide synthase gene, VdPKS1, whose mutation resulted in substantial virulence attenuation of $V$. dahlia (Zhang et al., 2017). In addition, baculoviruses infect insect species by invading into midgut epithelium of insect hosts. During the process, per os infectivity factors (PIFs) are critical in the infection. Wang et al. reviewed the advances in investigating the functions of PIFs, which would prompts in-depth study focused on the molecular interactions between insect and virus (Wang et al., 2017). Furthermore, Yin et al. investigated the effects of food supplementation on Brandt's vole population dynamics and plant community. Their results showed that food supplementation increased population density through increases in reproduction and survival during the non-breeding seasons, while survival during the breeding season significantly decreased due to the density-dependence effect (Yin et al., 2017).

It is our great honor to organize this thematic issue as guest-editors. Of course this issue did not include the entire picture of the representative work of research teams in the project, needless to say there are many other associated studies focusing on the plant-pest interactions in China. As the largest country accommodating about 1.4 billion people in the world, China will continue to support and reinforce this fascinating research area in the future, and we hope our project not only generates excellent achievements in science, but also fosters young and capable investigators to lead our future studies.

Compliance and ethics The author(s) declare that they have no conflict of interest.
Cao, J., Yu, Y., Huang, J., Liu, R., Chen, Y., Li, S., and Liu, J. (2017). Genome re-sequencing analysis uncovers pathogenecity-related genes undergoing positive selection in Magnaporthe oryzae. Sci China Life Sci 60, 880-890.

Chen, D., Chen, F., Chen, C., Chen, X., and Mao, Y. (2017). Transcriptome analysis of three cotton pests reveals features of gene expressions in the mesophyll feeder Apolygus lucorum. Sci China Life Sci 60, $826-838$.

Du, X., Wang, S., Gao, F., Zhang, L., Zhao, J., Guo, H., and Hua, C. (2017). Expression of pathogenesis-related genes in cotton roots in response to Verticillium dahliae PAMP molecules. Sci China Life Sci 60, $852-860$.

Guo, H., Wan, S., and Ge, F. (2017). Effect of elevated $\mathrm{CO}_{2}$ and $\mathrm{O}_{3}$ on phytohormone-mediated plant resistance to vector insects and insect-borne plant viruses. Sci China Life Sci 60, 816-825.

Guo, K., Yang, P., Chen, J., Lu, H., and Cui, F. (2017). Transcriptomic responses of three aphid species to chemical insecticide stress. Sci China Life Sci 60, 931-934.

Kan, J., Fang, R., and Jia, Y. (2017). Interkingdom signaling in plant-microbe interactions. Sci China Life Sci 60, 785-796.

Lai, Y., Chen, H., Wei, G., Wang, G., Li, F., and Wang, S. (2017). In vivo gene expression profiling of the entomopathogenic fungus Beauveria bassiana elucidates its infection stratagems in Anopheles mosquito. Sci China Life Sci 60, 839-851.

Sun, L., Qin, J., Wang, K., and Zhang, J. (2017). Expansion of pathogen recognition specificity in plants using pattern recognition receptors and artificially designed decoys. Sci China Life Sci 60, 797-805.

Wang, S., Zhou, F., Wang, B., Xu, D., Cao, Q., Lu, M., and Sun, J. (2017). Volatiles produced by bacteria alleviate antagonistic effects of one associated fungus on Dendroctonus valens larvae. Sci China Life Sci 60, 924-926.

Wang, W., Sun, Y., Han, L., Su, L., Xia, G., and Wang, H. (2017). Overexpression of GhPFN2 enhances protection against Verticillium dahliae invasion in cotton. Sci China Life Sci 60, 861-867.

Wang, X., Liu, X., Makalliwa, G.A., Li, J., Wang, H., Hu, Z., and Wang, M. (2017). Per os infectivity factors: a complicated and evolutionarily conserved entry machinery of baculovirus. Sci China Life Sci 60, 806-815.

Xiao, X., Cheng, X., Yin, K., Li, H., and Qiu, J. (2017). Abscisic acid negatively regulates post-penetration resistance of Arabidopsis to the biotrophic powdery mildew fungus. Sci China Life Sci 60, 891-901.

Yin, B., Li, G., Wan, X., Shang, G., Wei, W., and Zhang, Z. (2017). Large manipulative experiments reveal complex effects of food supplementation on population dynamics of Brandt's voles. Sci China Life Sci 60, 911-920.

Zhang, L., Lu, H., Guo, K., Yao, S., and Cui, F. (2017). Insecticide resistance status and detoxification enzymes of wheat aphids Sitobion avenae and Rhopalosiphum padi. Sci China Life Sci 60, 927-930.

Zhang, S., Ning, J., Zhang, W., Salcedo, C., and Zhao, L. (2017). Developmental differences between a Chinese and a North American isolate of the Pinewood Nematode Bursaphelenchus xylophilus (Tylenchida: Aphelenchoididae) under laboratory conditions. Sci China Life Sci 60, 921-923.

Zhang, T., Zhang, B., Hua, C., Meng, P., Wang, S., Chen, Z., Du, Y., Gao, F., and Huang, J. (2017). VdPKS1 is required for melanin formation and virulence in a cotton wilt pathogen Verticillium dahliae. Sci China Life Sci 60, 868-879.

Zhang, W., Meng, J., Ning, J., Qin, P., Zhou, J., Zou, Z., Wang, Y., Jiang, H., Ahmad, F., Zhao, L., and Sun, J. (2017). Differential immune responses of Monochamus alternatus against symbiotic and entomopathogenic fungi. Sci China Life Sci 60, 902-910. 


\section{Guest Editors' Biographical Sketch}

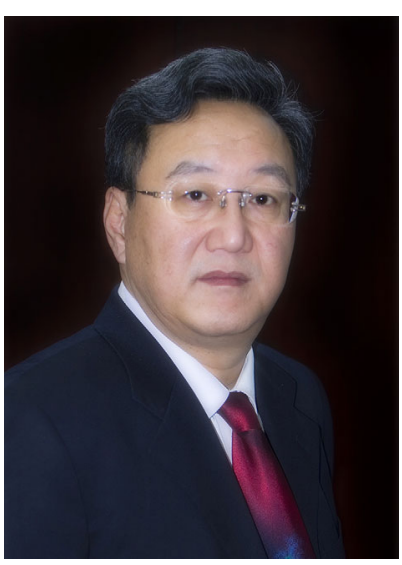

Dr. Le Kang is Distinguished Professor of the Institute of Zoology (IOZ), Chinese Academy of Sciences (CAS). He was elected as member of CAS in 2011 and member of the Developing World Academy of Sciences (TWAS) in 2012. Dr. Kang is an expert in entomology and ecological genomics. His work integrates multiple approaches from molecular biology, physiology to behavior analysis to resolve the ecological questions in insect adaptation to environmental variation and stress. He has authored about 160 international journals' papers, which were published in Science, PNAS, Nature Communications, PLoS Genetics, PLoS Pathogens, Genome Biology, Evolution, Molecular Ecology, Global Change Biology and so on. He has also been invited to write review articles for Annual Review of Entomology and Philosophical Transactions of the Royal Society. As a leading scientist, Dr. Kang is Editor-in-Chief of the international journal Insect Science, Associate Editor-in-Chief of Protein \& Cell and Zoological Research, and editorial board member of Journal of Insect Physiology, Current Opinion in Insect Science, Frontiers of Ecology and Evolution, and Science China Life Sciences.

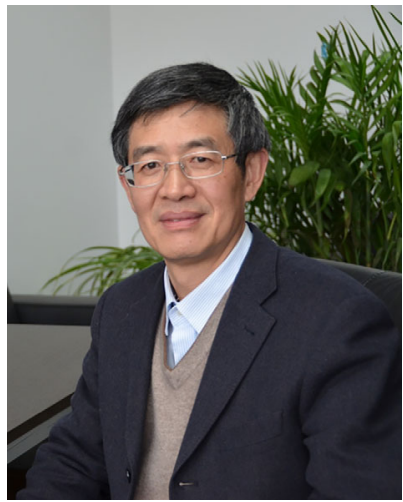

Dr. Xiao-Ya Chen is Professor in Institute of Plant Physiology and Ecology, Shanghai Institutes for Biological Sciences, Chinese Academy of Sciences (CAS). He was nominated as a CAS academician in 2005, the Developing World Academy of Sciences (TWAS) member in 2008. Currently he serves as the director of Shanghai Chenshan Plant Science Research Center, President of Chinese Society for Plant Biology (CSPB), Editor-in-Chief of Science Bulletin. Dr. Chen gained his BSc at Nanjing University, China, and Ph.D. at Reading University, UK. $\mathrm{He}$ is interested in plant secondary metabolism, plant-insect interactions and trichome (including cotton fiber) development. He and colleagues have conducted in-depth study of terpene biosynthesis and regulation in cotton, Artemisia annua, Arabidopsis thaliana, and Salvia spp. He has hitherto more than 100 publications, the majority of which in prestigious journals, including Nature Biotechnology, Nature Communications, Plant Cell, Molecular Plant, and PLoS Genetics.

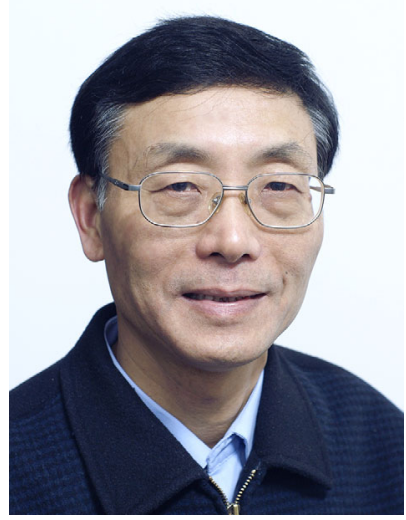

Dr. Rongxiang Fang, Senior Research Fellow of Institute of Microbiology, Member of Chinese Academy of Sciences (CAS), Member of the Developing World Academy of Sciences (TWAS). He has been engaged in basic and applied research in plant virology and plant biotechnology. Recently he is interested in the study of microbe-host interactions, including RNA silencing and suppression of RNA silencing, molecular mechanism of insect vectors in mediating plant diseases. He is the author of more than 150 manuscripts, which were published in academic journals such as Cell, Plant Cell, PNAS, PLoS Pathogens and Journal of Virology. 